\title{
Prevalence of urinary tract infections and antibiotics susceptibility pattern among infants and young children in Erbil city
}

\begin{tabular}{ccc}
\hline Chiman Hameed Saeed * & Kamal I. B. AL-Otraqchi $* *$ & Isam Y. Mansoor $* * *$ \\
\hline Abstract &
\end{tabular}

Background and objective: A urinary tract infection is defined by the presence of an organism in the urinary tract that is usually sterile. The age and gender are important factors influencing prevalence of urinary tract infections. The aim of this study was to clarify the microbiology of urinary tract infection, antibiotics susceptibility pattern prevalence and their relation with age, gender and circumcision among infants and young children in Erbil city.

Methods: Urine samples were collected from 300 children with sign and symptoms of urinary tract infection at Rapareen Pediatric Hospital in Erbil city. The specimens were cultured on appropriate media and the isolated organisms identified with a significant growth $10^{5} \mathrm{CFU} / \mathrm{ml}$. The susceptibility of bacteria causing urinary tract infection to different antimicrobial agents was investigated.

Results: The study showed that $43.3 \%$ of children had urinary tract infection $(53.2 \%$ females, $30.2 \%$ males). The highest percentage of infection occurred at age group 6-10 years with female's predominance. The percentage of urinary tract infections in uncircumcised boys was $76.9 \%$. The most common organism isolated was Escherichia coli $(33.8 \%)$. The isolated bacteria were highly sensitive to Ciprofloxacin, Amikacin and Nitrofurantoin.

Conclusion: Early and appropriate diagnosis of urinary tract infection in infants and young children is important as it is marker for urinary tract problems. Escherichia coli was the most common organism causing urinary tract infection in children. The most of urinary isolates had high level of resistance to commonly used antibiotics.

Keywords: UTIs, Circumcision, Bacteria, Candida, Antibiotics susceptibility.

\section{Introduction}

Urinary tract infections (UTI) are one of the most common and most series bacterial infections in children. It is estimated that at least $8 \%$ of girls and $2 \%$ of boys have UTI in childhood. ${ }^{1}$ The clinical signs and symptoms of UTI are non specific and vague in the first years of age. Such infection if untreated can lead to the subsequent renal scarring and are established risk factors of end stage renal disease, thus the high incidence of undiagnosed, improperly treated UTIs in young children is a cause of clinical and pubic concern. ${ }^{2}$ The incidence of UTI and its clinical impact are very different for both sexes and at different stages of life. It is known that UTI is more frequent in boys in the first three month of life, with sex distribution of 5:1 (male predominance). By preschool age the sex ratio is reversed with majority of UTI occurring in females. ${ }^{3}$ There is association between circumcision and UTI. It has been shown that the risk for UTI is increased, especially during the first year of life in uncircumcised infants. Uropathogenic colonization occurs to a greater extent around the meatus in uncircumcised children compared to circumcise ones in the first six months of life. ${ }^{4}$ Approximately

* Medical research centre, Hawler medical university, Erbil, Iraq.

** Department of microbiology, college of medicine, Hawler medical university, Erbil, Iraq.

$* * *$ Department of microbiology, college of Heath Science, Hawler medical university, Erbil, Iraq. 
$80 \%$ of urinary tract infections in children are caused by Escherichia coli followed by Klebsiella species, Proteus mirabilis, Pseudomonas aeruginosa and Enterobacter species, while $10 \%$ of UTIs are caused by Gram positive bacteria including Staphylococci, Enterococci and Streptococci. Fungus, such as Candida albicans is the second most common cause of nosocomial UTI in children and it can spread systemically. Fungi infections are seen in infants and children who are on long term antibiotics, patients who are immunocompromised, or patients using invasive devices like catheter. ${ }^{5}$ Bacteria causing urinary tract infections are grown resistance to antibiotics at high level. Antibiotic resistance complicates the treatment of urinary tract infection and is associated with a higher patient morbidity, higher costs of re-evaluation and re-treatment, higher rates of hospitalization and greater use of board-spectrum antibiotics. A population of organisms can lose its sensitivity to an antibiotic while the patient is under treatment. ${ }^{6}$ The aim of this study was to clarify the microbiology of urinary tract infection, antibiotics susceptibility pattern prevalence and their relation with age, gender and circumcision among infants and young children in Erbil city.

\section{Methods}

\section{Materials:}

Culture media: MacConkey agar (Oxoid), Blood agar (Rashmi Diagnostic), Muller Hinton agar (Rashmi Diagnostic), Sabouraud Dextrose agar (BD-Difco).

Reagents and solution: Catalase reagent $3 \%$ of $\left(\mathrm{H}_{2} \mathrm{O}_{2}\right)$, Oxidase reagent, Normal saline $(\mathrm{NaCl} 0.9 \%), \mathrm{HCL}$, Rabbit plasma, Human serum, Gram stain reagents.

Kits: API 20 E system, API STAPH, API 20 C AUX, Antibiotic discs.

During the period from October 2009 to March 2010 a total of three hundred urine samples were collected from infants and young children (inpatients and outpatients) aged one day -15 year, who were suffering from signs and symptoms of UTIs attending Rapareen Pediatric Hospital in Erbil city. The questionnaire used to assess the participants' health. It included questions about; age, gender, circumcision (for male), presence of sign and symptoms of UTI and previous infection (recurrent infection). All urine specimens were obtained aseptically by midstream cleancatch, catheterization or from urine bags. Ideally all significant cultures $\left(>10^{5} \mathrm{CFU} / \mathrm{ml}\right)$ were identified, pure growth of isolates were examined for the following morphology and biochemical characters:

1- Colony morphology.

2- Fermentation of sugar using API $20 \mathrm{E}$ system, API STAPH, API 20 C AUX.

3- Cell morphology and Gram reaction.

4- Catalase, Oxidase, Coagulase test.

5- Germ tube test for Candida albicans.

Disc diffusion method (Kirby- Bauermethod) was carried out according to the Clinical and Laboratory Standard institute guidelines (CLSI) was used for examine susceptibility of isolated bacteria to the following antibiotics: ampicillin (AMP), amoxicillin/ Clavulanic acid(AMC), penicillin $(P)$, gentamycin $(C N)$, sulfamethaxazoletrimethoprime (SXT), cephalexin $(C L)$, cephalothin(KF), nalidixic acid(NA), nitrofurantoin $(F)$, ciprofloxacin(CIP), ceftriaxone (CRO), chloramphenicol (C), amikacin(AK) and cefotaxime (CTX).

Statistical Analysis: Data analysis and calculation of $P$-value was done using the statistical package for the social sciences (version 16.0) through contingency coefficient test (CC test). A P value $\leq 0.05$ was regarded as statistically significant. This study was approved by the Research Ethics Committee of the College of Medicine, Hawler Medical University. 


\section{Results}

Of 300 urine samples, only 130 (43.3\%) showed culture positive. The percentage of UTIs in female and male were 91 $(53.2 \%)$ and 39 (30.2\%), respectively. The differences were highly significant (Table 1). In terms of age and gender, the highest percentage of infection $51(39.2 \%)$ occurred in the age group 6-10 year with female predominance, whereas males outnumbered females $14(60.9 \%)$ in the age group less than one year (Table 2). The relation between circumcision and UTIs are shown in the Figure1. The percentage of UTIs in uncircumcised boys $30(76.9 \%)$ was significantly higher than that of circumcised boys $9(23.1 \%)$. As shown in the Table 3, $50(38.5 \%)$ children had recurrent UTIs and their incidence in female $42(46.2 \%)$ was significantly higher than that of male $8(20.5 \%)$.

Table 1: Distribution of patients with urinary tract infection in relation to gender.

\begin{tabular}{|c|c|c|c|c|}
\hline \multirow[t]{2}{*}{ Gender } & & \multicolumn{2}{|c|}{ Culture results } & \multirow[t]{2}{*}{ Total } \\
\hline & & Positive & Negative & \\
\hline \multirow[t]{2}{*}{ Female } & No. & 91 & 80 & $\begin{array}{l}171 \\
57 \%\end{array}$ \\
\hline & $\%$ & $53.2 \%$ & $46.8 \%$ & \\
\hline \multirow[t]{2}{*}{ Male } & No. & 39 & 90 & $\begin{array}{l}129 \\
43 \%\end{array}$ \\
\hline & $\%$ & $30.2 \%$ & $69.8 \%$ & \\
\hline \multirow[t]{2}{*}{ Total } & No. & 130 & 170 & $\begin{array}{l}300 \\
100 \%\end{array}$ \\
\hline & $\%$ & $43.3 \%$ & $56.7 \%$ & \\
\hline
\end{tabular}

$P=<0.001$

Contingency Coefficient value for relation $=0.224$
Table 2: Incidence of UTI among male and female in relation to age and gender.

\begin{tabular}{lllllll}
\hline $\begin{array}{l}\text { Age } \\
\text { group } \\
\text { (Years) }\end{array}$ & \multicolumn{2}{l}{ Gender } & \multicolumn{3}{c}{ Female } & \multicolumn{3}{c}{ Male } & \multicolumn{2}{l}{ Total } \\
& No. & $\%$ & No. & $\%$ & No. & $\%$ \\
\hline$<1$ Year & 9 & $39.1 \%$ & 14 & $60.9 \%$ & 23 & $17.7 \%$ \\
$1-5$ & 30 & $73.2 \%$ & 11 & $26.8 \%$ & 41 & $31.5 \%$ \\
& & & & & & \\
$6-10$ & 42 & $82.4 \%$ & 9 & $17.6 \%$ & 51 & $39.2 \%$ \\
$11-15$ & 10 & $66.7 \%$ & 5 & $33.3 \%$ & 15 & $11.5 \%$ \\
& & & & & & \\
Total & 91 & $70.0 \%$ & 39 & $30.0 \%$ & 130 & $100.0 \%$
\end{tabular}

$P=0.002$

Contingency Coefficient value for relation $=0.316$

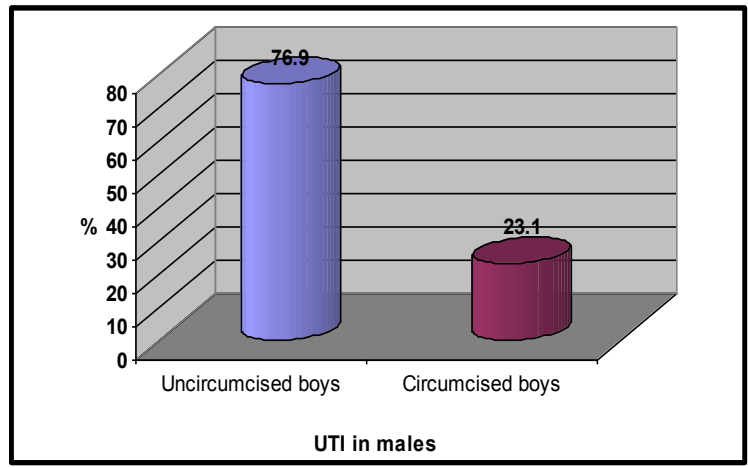

Figure 1: Incidence of UTI in relation to circumcision.

Table 3: The incidence of recurrent UTIs in relation to gender.

\begin{tabular}{|c|c|c|c|c|c|}
\hline \multirow{3}{*}{$\begin{array}{l}\text { Urinary } \\
\text { tract } \\
\text { infection }\end{array}$} & \multicolumn{4}{|c|}{ Gender } & \multirow{3}{*}{$\begin{array}{l}\text { Total } \\
\text { No. \% }\end{array}$} \\
\hline & \multicolumn{2}{|c|}{ Male } & \multicolumn{2}{|c|}{ Female } & \\
\hline & No. & $\%$ & No. & $\%$ & \\
\hline First & 31 & $79.5 \%$ & 49 & $53.8 \%$ & $80(61.5 \%)$ \\
\hline Recurrent & 8 & $20.5 \%$ & 42 & $46.2 \%$ & $50(38.5 \%)$ \\
\hline Total & 39 & $30 \%$ & 91 & $70 \%$ & $130(100 \%)$ \\
\hline
\end{tabular}


Figure 2 summarizes the isolated microorganisms, whereby the Gram negative bacteria $(68 \%)$ were more prevalent than Gram positive (26\%) and Candida albicans (6\%). Table 4 shows the percentage of isolated microorganisms in which $\mathrm{E}$. coli was the most common pathogen 48 $(33.8 \%)$ followed by Staphylococcus aureus $16(11.3 \%)$, all Klebsiella species $15(10.5 \%)$, Proteus mirabilis $14(9.9 \%)$, Pseudomonas aeruginosa $11(7.7 \%)$ and Candida albicans 8 (5.6\%).

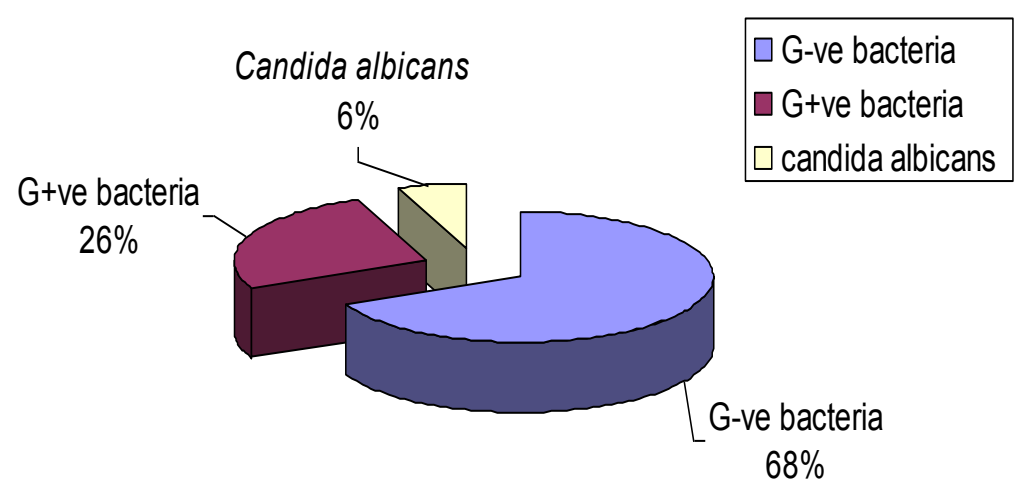

Figure 2: The total percentage of microorganisms isolated from positive urine culture.

Table 4: The number and percentage of uropathogens isolated from urine samples.

\begin{tabular}{lllllll}
\hline Isolated pathogens & \multicolumn{2}{l}{ Pure culture } & \multicolumn{2}{l}{ Mixed culture } & \multicolumn{2}{l}{ Total } \\
& No. & $\%$ & No. & $\%$ & No. & $\%$ \\
\hline Escherichia coli & 40 & $33.9 \%$ & 8 & $33.3 \%$ & 48 & $33.8 \%$ \\
Klebsiella pneumonia & 6 & $5.1 \%$ & 1 & $4.2 \%$ & 7 & $4.9 \%$ \\
Klebsiella oxytoca & 4 & $3.4 \%$ & 2 & $8.3 \%$ & 6 & $4.2 \%$ \\
Klebsiella terrigena & 1 & $0.8 \%$ & 0 & $0 \%$ & 1 & $0.7 \%$ \\
Klebsiella ornithinolytica & 1 & $0.8 \%$ & 0 & $0 \%$ & 1 & $0.7 \%$ \\
Proteus mirabilis & 11 & $9.3 \%$ & 3 & $12.5 \%$ & 14 & $9.9 \%$ \\
Pseudomonas aeruginosa & 8 & $6.8 \%$ & 3 & $12.5 \%$ & 11 & $7.7 \%$ \\
Enterobacter aerogenes & 5 & $4.2 \%$ & 0 & $0 \%$ & 5 & $3.5 \%$ \\
Staphylococcus aureus & 16 & $13.6 \%$ & 0 & $0 \%$ & 16 & $11.3 \%$ \\
Staphylococcus epidermidis & 7 & $5.9 \%$ & 0 & $0 \%$ & 7 & $4.9 \%$ \\
Staphylococcus saprophyticus & 2 & $1.7 \%$ & 1 & $4.2 \%$ & 3 & $2.1 \%$ \\
Staphylococcus hemolyticus & 5 & $4.2 \%$ & 0 & $0 \%$ & 5 & $3.5 \%$ \\
Staphylococcus xylosus & 3 & $2.5 \%$ & 1 & $4.2 \%$ & 4 & $2.8 \%$ \\
Staphylococcus lugdunensis & 1 & $0.8 \%$ & 0 & $0 \%$ & 1 & $0.7 \%$ \\
Enterococcus spp. & 5 & $4.2 \%$ & 0 & $0 \%$ & 5 & $3.5 \%$ \\
Candida albicans & 3 & $2.5 \%$ & 5 & $20.8 \%$ & 8 & $5.6 \%$ \\
Total & 118 & $83.1 \%$ & 24 & $16.9 \%$ & 142 & $100 \%$ \\
\hline \hline
\end{tabular}


The results of susceptibility of Gram negative and Gram positive bacteria to antibiotics are presented in Table 5 and Table 6 , respectively. The isolated bacteria were highly sensitive to amikacin, nitrofurantoin and ciprofloxacin and resistant to penicillin, ampicillin, sulfamethaxazole-trimethoprime, cephalothin, ceftriaxone and cefotaxime sodium.

Table 5: The percentage of antibiotics resistance in Gram negative bacteria.

\begin{tabular}{|c|c|c|c|c|c|c|c|c|c|c|c|c|c|c|}
\hline $\begin{array}{l}\text { Isolated } \\
\text { bacteria } \\
\& \text { No. }\end{array}$ & AMP & AMC & $\mathbf{P}$ & CN & SXT & C & CL & CTX & CRO & KF & $F$ & CIP & AK & NA \\
\hline $\begin{array}{l}\text { Escherichia } \\
\text { coli (48) }\end{array}$ & $\begin{array}{l}46 \\
95.8 \%\end{array}$ & $\begin{array}{l}41 \\
85.4 \%\end{array}$ & $\begin{array}{l}48 \\
100 \%\end{array}$ & $\begin{array}{l}28 \\
58.3 \%\end{array}$ & $\begin{array}{l}42 \\
87.5 \%\end{array}$ & $\begin{array}{l}14 \\
29.2 \%\end{array}$ & $\begin{array}{l}34 \\
70.8 \%\end{array}$ & $\begin{array}{l}39 \\
81.3 \%\end{array}$ & $\begin{array}{l}36 \\
75 \%\end{array}$ & $\begin{array}{l}42 \\
87.5 \%\end{array}$ & $\begin{array}{l}7 \\
14.6 \%\end{array}$ & $\begin{array}{l}10 \\
20.8 \%\end{array}$ & $\begin{array}{l}3 \\
6.3 \%\end{array}$ & $\begin{array}{l}29 \\
60.4 \%\end{array}$ \\
\hline $\begin{array}{l}\text { Klebsiella } \\
\text { pneumoniae } \\
\text { (7) }\end{array}$ & $\begin{array}{l}7 \\
100 \%\end{array}$ & $\begin{array}{l}4 \\
57.1 \%\end{array}$ & $\begin{array}{l}7 \\
100 \%\end{array}$ & $\begin{array}{l}5 \\
71.4 \%\end{array}$ & $\begin{array}{l}7 \\
100 \%\end{array}$ & $\begin{array}{l}1 \\
14.3 \%\end{array}$ & $\begin{array}{l}7 \\
100 \%\end{array}$ & $\begin{array}{l}7 \\
100 \%\end{array}$ & $\begin{array}{l}3 \\
42.9 \%\end{array}$ & $\begin{array}{l}7 \\
100 \%\end{array}$ & $\begin{array}{l}3 \\
42.9 \%\end{array}$ & $\begin{array}{l}0 \\
0 \%\end{array}$ & $\begin{array}{l}4 \\
57.1 \%\end{array}$ & $\begin{array}{l}4 \\
57.1 \%\end{array}$ \\
\hline $\begin{array}{l}\text { Klebsiella } \\
\text { oxytoca (6) }\end{array}$ & $\begin{array}{l}1 \\
16.7 \%\end{array}$ & $\begin{array}{l}1 \\
16.7 \%\end{array}$ & $\begin{array}{l}6 \\
100 \%\end{array}$ & $\begin{array}{l}3 \\
50 \%\end{array}$ & $\begin{array}{l}2 \\
33.3 \%\end{array}$ & $\begin{array}{l}5 \\
83.3 \%\end{array}$ & $\begin{array}{l}1 \\
16.7 \%\end{array}$ & $\begin{array}{l}2 \\
33.3 \%\end{array}$ & $\begin{array}{l}1 \\
16.7 \%\end{array}$ & $\begin{array}{l}0 \\
0 \%\end{array}$ & $\begin{array}{l}3 \\
50 \%\end{array}$ & $\begin{array}{l}5 \\
83.3 \%\end{array}$ & $\begin{array}{l}6 \\
100 \%\end{array}$ & $\begin{array}{l}5 \\
83.3 \%\end{array}$ \\
\hline $\begin{array}{l}\text { Klebsiella } \\
\text { terrigena (1) }\end{array}$ & $\begin{array}{l}1 \\
100 \%\end{array}$ & $\begin{array}{l}1 \\
100 \%\end{array}$ & $\begin{array}{l}1 \\
100 \%\end{array}$ & $\begin{array}{l}1 \\
100 \%\end{array}$ & $\begin{array}{l}1 \\
100 \%\end{array}$ & $\begin{array}{l}0 \\
0 \%\end{array}$ & $\begin{array}{l}1 \\
100 \%\end{array}$ & $\begin{array}{l}1 \\
100 \%\end{array}$ & $\begin{array}{l}1 \\
100 \%\end{array}$ & $\begin{array}{l}1 \\
100 \%\end{array}$ & $\begin{array}{l}1 \\
100 \%\end{array}$ & $\begin{array}{l}0 \\
0 \%\end{array}$ & $\begin{array}{l}0 \\
0 \%\end{array}$ & $\begin{array}{l}1 \\
100 \%\end{array}$ \\
\hline $\begin{array}{l}\text { Klebsiella } \\
\text { ornithinolytica } \\
\text { (1) }\end{array}$ & $\begin{array}{l}1 \\
100 \%\end{array}$ & $\begin{array}{l}0 \\
0 \%\end{array}$ & $\begin{array}{l}1 \\
100 \%\end{array}$ & $\begin{array}{l}1 \\
100 \%\end{array}$ & $\begin{array}{l}1 \\
100 \%\end{array}$ & $\begin{array}{l}0 \\
0 \%\end{array}$ & $\begin{array}{l}1 \\
100 \%\end{array}$ & $\begin{array}{l}1 \\
100 \%\end{array}$ & $\begin{array}{l}0 \\
0 \%\end{array}$ & $\begin{array}{l}1 \\
100 \%\end{array}$ & $\begin{array}{l}0 \\
0 \%\end{array}$ & $\begin{array}{l}0 \\
0 \%\end{array}$ & $\begin{array}{l}1 \\
100 \%\end{array}$ & $\begin{array}{l}1 \\
100 \%\end{array}$ \\
\hline $\begin{array}{l}\text { Proteus } \\
\text { mirabilis (14) }\end{array}$ & $\begin{array}{c}12 \\
85.7 \%\end{array}$ & $\begin{array}{c}9 \\
64.3 \%\end{array}$ & $\begin{array}{c}12 \\
100 \%\end{array}$ & $\begin{array}{l}10 \\
71.4 \%\end{array}$ & $\begin{array}{l}13 \\
92.9 \%\end{array}$ & $\begin{array}{l}9 \\
64.3 \%\end{array}$ & $\begin{array}{l}12 \\
85.7 \%\end{array}$ & $\begin{array}{l}8 \\
57.1 \%\end{array}$ & $\begin{array}{l}9 \\
64.3 \%\end{array}$ & $\begin{array}{l}12 \\
85.7 \%\end{array}$ & $\begin{array}{l}7 \\
50 \%\end{array}$ & $\begin{array}{l}5 \\
35.7 \%\end{array}$ & $\begin{array}{l}2 \\
14.3 \%\end{array}$ & $\begin{array}{l}9 \\
64.3 \%\end{array}$ \\
\hline $\begin{array}{l}\text { Pseudo monas } \\
\text { aeruginosa } \\
\text { (11) }\end{array}$ & $\begin{array}{c}9 \\
81.8 \%\end{array}$ & $\begin{array}{c}10 \\
90.9 \%\end{array}$ & $\begin{array}{c}11 \\
100 \%\end{array}$ & $\begin{array}{l}2 \\
18.2 \%\end{array}$ & $\begin{array}{l}6 \\
54.5 \%\end{array}$ & $\begin{array}{l}5 \\
45.5 \%\end{array}$ & $\begin{array}{l}10 \\
90.9 \%\end{array}$ & $\begin{array}{l}9 \\
81.8 \%\end{array}$ & $\begin{array}{l}8 \\
72.7 \%\end{array}$ & $\begin{array}{l}7 \\
63.6 \%\end{array}$ & $\begin{array}{l}7 \\
63.6 \%\end{array}$ & $\begin{array}{l}2 \\
18.2 \%\end{array}$ & $\begin{array}{l}0 \\
0 \%\end{array}$ & $\begin{array}{l}9 \\
81.8 \%\end{array}$ \\
\hline $\begin{array}{l}\text { Enterobacter } \\
\text { aerogenes (5) }\end{array}$ & $\begin{array}{c}5 \\
100 \%\end{array}$ & $\begin{array}{c}3 \\
60 \%\end{array}$ & $\begin{array}{c}5 \\
100 \%\end{array}$ & $\begin{array}{l}1 \\
20 \%\end{array}$ & $\begin{array}{l}4 \\
80 \%\end{array}$ & $\begin{array}{l}2 \\
40 \%\end{array}$ & $\begin{array}{l}3 \\
60 \%\end{array}$ & $\begin{array}{l}5 \\
100 \%\end{array}$ & $\begin{array}{c}4 \\
80 \%\end{array}$ & $\begin{array}{l}5 \\
100 \%\end{array}$ & $\begin{array}{l}1 \\
20 \%\end{array}$ & $\begin{array}{l}2 \\
40 \%\end{array}$ & $\begin{array}{l}0 \\
0 \%\end{array}$ & $\begin{array}{c}4 \\
80 \%\end{array}$ \\
\hline
\end{tabular}

Table (6): The percentage of antibiotics resistance in Gram positive bacteria.

\begin{tabular}{|c|c|c|c|c|c|c|c|c|c|c|c|c|c|c|}
\hline $\begin{array}{l}\text { Isolated } \\
\text { bacteria } \\
\& \text { No. }\end{array}$ & AMP & AM & $\mathbf{P}$ & $\mathrm{CN}$ & SXT & C & $C L$ & СтХ & CRO & KF & $F$ & CIP & AK & NA \\
\hline $\begin{array}{l}\text { Staphylococcus } \\
\text { aureus (16) }\end{array}$ & $\begin{array}{l}13 \\
81.3 \%\end{array}$ & $\begin{array}{l}12 \\
75 \%\end{array}$ & $\begin{array}{l}16 \\
100 \%\end{array}$ & $\begin{array}{l}6 \\
37.5 \%\end{array}$ & $\begin{array}{l}11 \\
68.8 \%\end{array}$ & $\begin{array}{l}12 \\
75 \%\end{array}$ & $\begin{array}{l}4 \\
25 \%\end{array}$ & $\begin{array}{l}14 \\
87.5 \%\end{array}$ & $\begin{array}{l}12 \\
75 \%\end{array}$ & $\begin{array}{l}5 \\
31.3 \%\end{array}$ & $\begin{array}{l}0 \\
0 \%\end{array}$ & $\begin{array}{l}2 \\
12.5 \%\end{array}$ & $\begin{array}{l}0 \\
0 \%\end{array}$ & $\begin{array}{l}5 \\
31.3 \%\end{array}$ \\
\hline $\begin{array}{l}\text { Staphylococcus } \\
\text { hemolyticus(5) }\end{array}$ & $\begin{array}{l}4 \\
80 \%\end{array}$ & $\begin{array}{l}4 \\
80 \%\end{array}$ & $\begin{array}{l}5 \\
100 \%\end{array}$ & $\begin{array}{l}2 \\
40 \%\end{array}$ & $\begin{array}{l}4 \\
80 \%\end{array}$ & $\begin{array}{l}3 \\
60 \%\end{array}$ & $\begin{array}{l}4 \\
80 \%\end{array}$ & $\begin{array}{l}4 \\
80 \%\end{array}$ & $\begin{array}{l}3 \\
60 \%\end{array}$ & $\begin{array}{l}1 \\
20 \%\end{array}$ & $\begin{array}{l}0 \\
0 \%\end{array}$ & $\begin{array}{l}2 \\
40 \%\end{array}$ & $\begin{array}{l}1 \\
20 \%\end{array}$ & $\begin{array}{l}2 \\
40 \%\end{array}$ \\
\hline $\begin{array}{l}\text { Staphylococcus } \\
\text { xylosus (4) }\end{array}$ & $\begin{array}{l}2 \\
50 \%\end{array}$ & $\begin{array}{l}3 \\
75 \%\end{array}$ & $\begin{array}{l}4 \\
100 \%\end{array}$ & $\begin{array}{l}3 \\
75 \%\end{array}$ & $\begin{array}{l}4 \\
100 \%\end{array}$ & $\begin{array}{l}4 \\
100 \%\end{array}$ & $\begin{array}{l}1 \\
25 \%\end{array}$ & $\begin{array}{l}3 \\
75 \%\end{array}$ & $\begin{array}{l}2 \\
50 \%\end{array}$ & $\begin{array}{l}3 \\
75 \%\end{array}$ & $\begin{array}{l}0 \\
0 \%\end{array}$ & $\begin{array}{l}1 \\
25 \%\end{array}$ & $\begin{array}{l}1 \\
25 \%\end{array}$ & $\begin{array}{l}1 \\
25 \%\end{array}$ \\
\hline $\begin{array}{l}\text { Staphylococcus } \\
\text { saprophyticus(3) }\end{array}$ & $\begin{array}{l}1 \\
33.3 \%\end{array}$ & $\begin{array}{l}3 \\
100 \%\end{array}$ & $\begin{array}{l}3 \\
100 \%\end{array}$ & $\begin{array}{l}0 \\
0 \%\end{array}$ & $\begin{array}{l}2 \\
66.7 \%\end{array}$ & $\begin{array}{l}2 \\
66.7 \%\end{array}$ & $\begin{array}{l}0 \\
0 \%\end{array}$ & $\begin{array}{l}3 \\
100 \%\end{array}$ & $\begin{array}{l}3 \\
100 \%\end{array}$ & $\begin{array}{l}0 \\
0 \%\end{array}$ & $\begin{array}{l}0 \\
0 \%\end{array}$ & $\begin{array}{l}0 \\
0 \%\end{array}$ & $\begin{array}{l}1 \\
33.3 \%\end{array}$ & $\begin{array}{l}1 \\
33.3 \%\end{array}$ \\
\hline $\begin{array}{l}\text { Staphylococcus } \\
\text { epidermidis (7) }\end{array}$ & $\begin{array}{l}4 \\
57.1 \%\end{array}$ & $\begin{array}{l}4 \\
57.1 \%\end{array}$ & $\begin{array}{l}5 \\
71.4 \%\end{array}$ & $\begin{array}{l}4 \\
57.1 \%\end{array}$ & $\begin{array}{l}5 \\
71.4 \%\end{array}$ & $\begin{array}{l}6 \\
85.7 \%\end{array}$ & $\begin{array}{l}2 \\
28.6 \%\end{array}$ & $\begin{array}{l}6 \\
85.7 \%\end{array}$ & $\begin{array}{l}6 \\
85.7 \%\end{array}$ & $\begin{array}{l}1 \\
14.3 \%\end{array}$ & $\begin{array}{l}0 \\
0 \%\end{array}$ & $\begin{array}{l}1 \\
14.3 \%\end{array}$ & $\begin{array}{l}2 \\
28.6 \%\end{array}$ & $\begin{array}{l}2 \\
28.6 \%\end{array}$ \\
\hline $\begin{array}{l}\text { Staphylococcus } \\
\text { lugdunesis(1) }\end{array}$ & $\begin{array}{l}0 \\
0 \%\end{array}$ & $\begin{array}{l}1 \\
100 \%\end{array}$ & $\begin{array}{l}1 \\
100 \%\end{array}$ & $\begin{array}{l}1 \\
100 \%\end{array}$ & $\begin{array}{l}0 \\
100 \%\end{array}$ & $\begin{array}{l}1 \\
100 \%\end{array}$ & $\begin{array}{l}0 \\
0 \%\end{array}$ & $\begin{array}{l}1 \\
100 \%\end{array}$ & $\begin{array}{l}1 \\
100 \%\end{array}$ & $\begin{array}{l}1 \\
100 \%\end{array}$ & $\begin{array}{l}0 \\
0 \%\end{array}$ & $\begin{array}{l}1 \\
100 \%\end{array}$ & $\begin{array}{l}0 \\
0 \%\end{array}$ & $\begin{array}{l}0 \\
0 \%\end{array}$ \\
\hline $\begin{array}{l}\text { Enterococcus } \\
\text { spp. (5) }\end{array}$ & $\begin{array}{l}1 \\
20 \%\end{array}$ & $\begin{array}{l}3 \\
60 \%\end{array}$ & $\begin{array}{l}1 \\
20 \%\end{array}$ & $\begin{array}{l}1 \\
20 \%\end{array}$ & $\begin{array}{l}4 \\
80 \%\end{array}$ & $\begin{array}{l}3 \\
60 \%\end{array}$ & $\begin{array}{l}0 \\
100 \%\end{array}$ & $\begin{array}{l}2 \\
40 \%\end{array}$ & $\begin{array}{l}1 \\
20 \%\end{array}$ & $\begin{array}{l}3 \\
60 \%\end{array}$ & $\begin{array}{l}0 \\
0 \%\end{array}$ & $\begin{array}{l}1 \\
20 \%\end{array}$ & $\begin{array}{l}2 \\
40 \%\end{array}$ & $\begin{array}{l}3 \\
60 \%\end{array}$ \\
\hline
\end{tabular}




\section{Discussion}

Urinary tract infection in children is significant source of morbidity. It is noted that the risk of renal damage from UTI is greatest in children younger than 5 years; thus early diagnosis and prompt treatment are important. $^{7}$ Freedman $^{8}$ found that the infection of urinary tract is relatively common in children, accounting for a significant number of pediatric hospital admissions. In the present study the percentage of UTIs among children was $43.3 \%$. Similar results were reported by other investigators ${ }^{9}$ who reported that $45.2 \%$ of examined children patients were confirmed as having UTI. The percentage of UTI in females $(53.2 \%)$ was higher than that of males $(30.2 \%)$. These results are consistent with obtained in a study done by AL-Momani ${ }^{10}$ who found that prevalence of UTI among females was higher than males (59\% females, $41 \%$ males respectively). Females may get UTI from the bowel due to shortness of the female urethra and its close location to the perineal region. Thus, the organisms ascend to the urinary tract. ${ }^{11}$ In this study the UTIs was distributed between age and gender and the high incidence of infection occurred at age group 6-10 years $(39.2 \%)$ in which was significantly higher in female than that of male. This observation was in accordance with finding of Abdulhadi, ${ }^{12}$ Bay and Anacleto. ${ }^{3}$ The high incidence of infection in these age groups, especially among girls may due to poor perineal hygiene, errors in self management, errors in management made by schoolgirls are to clean the perineum forwards from the anus to the valuva and to micturate infrequently. Infrequent micturition leads to over distension of the bladder, with subsequent incomplete emptying; residual urine then interferes with bladder defenses by allowing bacterial colonization of bladder urine to occur more readily. ${ }^{13}$ It is known that UTI is more frequent in boys in the first three month of life. In the present study this was also true as the percentage of UTIs was significantly higher in male $(60.9 \%)$ than female
$(39.1 \%)$. This very close to the value arrived at in the study done by Rehman and coworkers ${ }^{14}$ who reported that prevalence of UTI in male children (50\%) was found to be higher than female in age group less than one year and declined gradually with increasing age. During neonatal period and the first months of life there is marked male to female preponderance, may be due to congenital obstruction, phimosis and high frequency of urethral malformation. ${ }^{15}$ The frequency of UTI in uncircumcised boys (76.9\%) estimated in this study and was significantly higher than that of circumcised boys $(23.1 \%)$. Ali and $\mathrm{Osman}^{9}$ in their study reported that UTI was more prevalent among uncircumcised boys. Aartan and Coworkers ${ }^{16}$ documented that uncircumcised boys are 10 times more likely to have urinary tract infection during their first of life than circumcised boys and they added that the preputial sac can acts as a reservoir of organisms and is thus responsible for causing ascending UTI and contamination by enteric flora in the first 6 years of life is especially likely to occur while using diapers. The results showed that $38.5 \%$ of children had recurrent UTI and such infection was significantly higher in female $(46.2 \%)$ than that of male $(20.5 \%)$. Taneja and colleagues ${ }^{17}$ found that recurrent UTI among children was $71.6 \%$. Barroso and coworkers ${ }^{18}$ stated that recurrent UTI is frequent and occurs in approximately $40 \%$ in females and $32 \%$ in males. There might be a number of most probable explanations for these findings including that the patients are treated without true identification of causative organism, wrong antibiotics have been used, insufficient dosing, development of bacterial resistance to antibiotic or structural abnormalities of the urinary tract and low immunity. ${ }^{19} \mathrm{E}$. coli was the most prevalent organism isolated in this study. Our result agrees with that obtained by Jombo and coworkers ${ }^{20}$ who found that $\mathrm{E}$. coli was the most uropathogen identified at rate $36.4 \%$. The primary virulence 
factors associated with the ability of $\mathrm{E}$. coli to cause UTIs is pili, which allow uropathogic strain to adhere to epithelial cells and not be washed out with urine flow. ${ }^{21}$ The study also showed that the percentage of UTIs caused by Candida ablicans was $5.6 \%$. This result was close to the finding of Taneja. ${ }^{17}$ Anbumani and Mallika ${ }^{22}$ found that amikacin and nitrofurantoin showed good activity against uropathogen isolates. These results are in agreement with that obtained in present study. In present study the result showed that the most of uropathogens isolates had very high degree of resistance to penicillin, ampicillin, sulfamethaxazole-trimethoprime, cephalothin, ceftriaxone and cefotaxime sodium. Ranjbar and coworker ${ }^{23}$ concluded that the most of urinary isolates had a high level of resistance to commonly used antibiotics. Susceptibility to antibiotics is changing in general and increase in antibiotic resistance has been shown worldwide. The main reason for this trend is the increase in antibiotic consumption, the abuse of board spectrum antibiotics or self medication. ${ }^{24}$

\section{Conclusion}

Early and appropriate diagnosis of UTI in infants and young children is important as it is marker for urinary tract problems such as renal scarring and reflux nephropathy which can lead to hypertension and end stage renal failure. The rate of UTI was higher in male children at early ages compared to that of female however; the rate of infection seems to increase in female, as they get older. The incidence of recurrent UTI was more common among female than male. E. coli was the most common organism causing UTI in children and Candida albicans seems to be another important agent causing UTI in children. The emergency of multiple drugs resistance strains is worrying and the use of antibiotics should not be random.

\section{Conflicts of interest}

The author reports no conflicts of interest.

\section{References}

1. Spencer JD, Schwadere A, McHuge K, Hanis DS. Pediatric urinary tract infections: an analysis of hospitalizations, charge, and costs in the USA. Pediatr Nephrol 2010; 25: 2469-75.

2. Shaikh N, Morone NE, Farrel MH. Prevalence of urinary tract infection in children. A metaanalysis. Pediatr Infect Dis J 2008; 27(4):302-8.

3. Bay AG, Anacleto F. Clinical and laboratory profile of urinary tract infection in children among children at outpatient clinic of a Tertiary Hospital. PIDSPJ 2010; 11(1):10-6.

4. Berk B, Ozgu A, Semih T, Tarkan S. Circumcision: Pros and cons. Indian J Urol 2010; 26:12-5.

5. Rai GK, Uperti HC. Rai SK, Shan KP Shrestha RM. Causative agents of urinary tract infections in children and their antibiotic sensitivity pattern: a hospital based study. Nepal Med Coll J 2008; 10 (2):86-90.

6- Wagenlehner F Naber K. Treatment of bacterial urinary tract infections: presence and future. Eur Urol 2006; 49:235-44.

7. Mohammed W, Abu G Anahtar K. Screening test for detection of urinary tract infections: Evolution of urinary leukocytes esterase dipstick test. TAF Prev Med Bull 2008; 7:187-90.

8. Freedman A. Urologic diseases in North America project: trends in resource utilization for urinary tract infection in children. J urol 2005; 173:949-54.

9. Ali EM A, Osman AH. Acute urinary tract infections in children in Khartoum State: pathogens, antimicrobial susceptibility and associated risk factors. Arab Journal of Nephrology and Transplantation 2009; 2(2):11-5.

10. Al-Momani T. Microbiological study of urinary tract infection in children at Princess Haya Hospital in South of Jordan. MEJFM 2006; 4(2):3-7.

11. Al-Haddad A M. Urinary tract infection among pregnant women in Al-Mukalla district. Yemen. EMHJ 2005; 11(3): 505-10.

12. Abdulhadi SK, Yashua AH, Uba A. Organisms causing urinary tract infection in pediatric patients at Murtala Muhammad Specialist Hospital, Kano Nigeria. International Journal of Biomedical and Health Sciences 2008; 4(4):165-7.

13. Modarres S, Oskoii NN. Bacterial etiologic agents of urinary tract infections in children in the Islamic Republic of Iran. EMHJ 1997; 3(2):290-5.

14. Rehman AU, Jahanzeb M, Siddiqui TS, Idris M. Frequency and clinical presentation of UTI among children of Hazara division. Pakistan J Ayub Med Coll Abbottabab 2008; 20(1): 63-5.

15. Theodoros A, Kanelloponlo S, Salakos C, Spilliopoulou I, Ellina A. Nikolakopoulou N M, et al. First urinary infection in neonate, infants and young children: a comparative study. Pediatric Nephrol 2006; 21:1131-7.

16. Agartan CA, Kaya DA, Ozkurk CE, Gulcan A. Is aerobic perputial flora age dependent?. JPN J Infect Dis 2005; 58: 276-8. 
17. Taneja N, Chatterjee SS, Singh M SS Sharma M. Pediatric urinary tract infection in a tertiary care center from north India. Indian J Med Res 2010; 131:101-5.

18. Barroso U, Barroso DV, Jacobino M, Vinhaes AJ, Macedo A, Srougi M. Etiology of urinary tract infection in scholor children. International Braz J Urol 2003; 29(5):450- 4.

19. Thomas M, Hooton TM. Recurrent urinary tract infection in women. Intern. J Ant Agen 2001; 17:259-68.

20. Jombo GTA, Odey F, Ibor S, Bolarin DM, Ejezie GC, Egah DZ. Subclinical significant bacteriuria among preschool children in Calabar Municipality: A survey. J Med Sci 2010; 1(4):134-40.

21. Amrilak I, Amrilak B. Urinary tract infection in different pediatric age groups: an overview of diagnosis, investigation, management and outcome. Int Pediatr 2007; 22 (3):137-49.

22. Anbumani N, Mallika M. Antibiotic resistance pattern in uropathogens in a Tertiary Care Hospital. Indmedica- Indian Journal for the Practising Doctor 2007; 4 (1): 1-8.

23. Ranjbar R, Haghi- Ashtiani MT, Jonaidi Jafari N, Abedini M. The prevalence and antimicrobial susceptibility of bacterial uropathogens isolated from pediatric patients. Iranian J Pub Health 2009; $38(2): 134-8$.

24. Younis N, Quaol K, Al-Momani T, Al-Awaisheh F, Al-Kayed D. Antibiotic resistance in children with recurrent or complicated urinary tract infection. J Nepal Med Assoc 2009; 48(173):14-9. 